\title{
Molecular Vibration Accelerates Charge Transfer \\ Emission in a Highly Twisted Blue Thermally Activated Delayed Fluorescence Material
}

Yoshimasa Wada, Katsuyuki Shizu, and Hironori Kaji*

Institute for Chemical Research, Kyoto University, Uji, Kyoto 611-0011, Japan

*E-mail: kaji@scl.kyoto-u.ac.jp 


\section{General procedure for the calculation}

FCHT calculation was performed using Gaussian 16. $S_{0}$ and $S_{1}$ optimized structures were used for the calculation after the frequency analysis. All vibronic transitions contributing equal to or greater than $0.001 \%$ to the total intensity of the spectrum are provided in the Figure 2, 3, S3 and S4. The number of contributing modes ( $N_{\text {modes }}$ ) are shown in Figure $\mathrm{S} 4$. Contribution of $\mathrm{C}_{1}, \mathrm{C}_{2}, \mathrm{C}_{3}$, and $\mathrm{C}_{4}$ to the total emission intensity were comparable as mentioned in the manuscript. This is because $\mu^{2}$ values of modes in $\mathrm{C}_{1}$ are stronger than those in $\mathrm{C}_{2}, \mathrm{C}_{3}$, and $\mathrm{C}_{4}$, while smaller $N_{\text {modes }}$ in $\mathrm{C}_{1}$ than those in $\mathrm{C}_{2}, \mathrm{C}_{3}$, and $\mathrm{C}_{4}$. The total valance between absolute $\mu^{2}$ values and $N_{\text {modes }}$ result in the comparable contribution of $\mathrm{C}_{1}, \mathrm{C}_{2}, \mathrm{C}_{3}$, and $\mathrm{C}_{4}$.

\section{Calculation at the M06-2X/6-31G(d) level of theory}

The scaling factor for the vibrational frequency was 0.947 according to the database on NIST ${ }^{1}$. Calculated vibronic spectrum was shown in the Figure S5 (green) together with that of PBE0/6-31G(d) (black). Although the relative intensity of vibronic progressions differs from each other, the overall shape including peak positions agrees well. Figure S6 displays vibrational modes exhibiting strong transition dipole moment in $\mathrm{C}_{1}$ and $\mu^{2}$ values of vibronic transitions in $\mathrm{C}_{1}$ as a function of vibrational frequency. Although there can be seen differences of mode numbers ("Mode 8" and "Mode 91") compared with those of PBE0/6-31G(d) calculation (Figure 3 and Figure S2), these modes correspond to "Mode 9" and "Mode 90" in PBE0/6-31G(d) calculation, respectively. So, all vibrational modes providing strong transition dipole moment are consistent. In addition, the difference of vibrational frequency was found to be very small between those different functionals.

\section{Calculation of anthracene as an example of aromatic molecules}

FCHT calculation was conducted on the anthracene at the PBE0/6-31G(d) level of theory. The symmetry was $D_{2 \mathrm{~h}}$. Figure $\mathrm{S} 7$ shows the mode $48\left(1404 \mathrm{~cm}^{-1}\right)$ exhibiting the strongest transition dipole moment in $\mathrm{C}_{1}$. Scaling factor for the vibrational frequency was 0.95 according to the database on NIST ${ }^{1}$. 


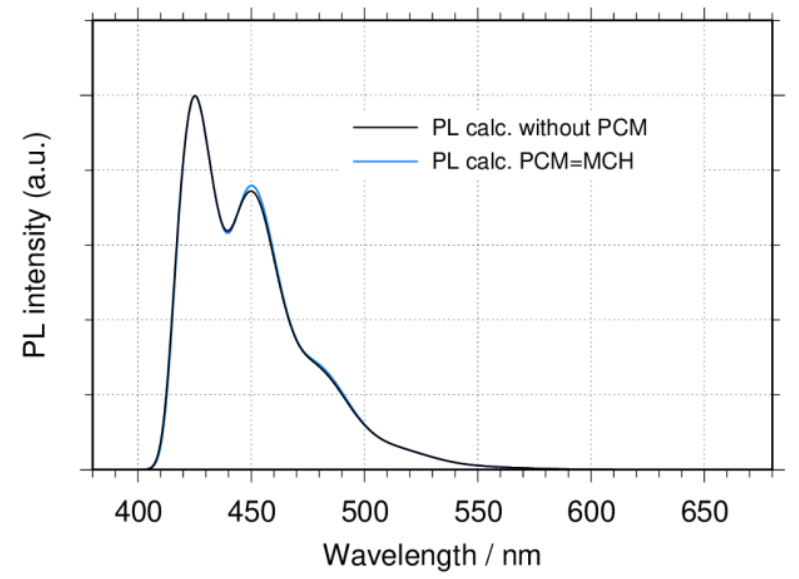

Figure S1. Calculated vibronic spectra of MA-TA for $S_{1}-S_{0}$ transition using PBE0/6-31G(d) without (black) and with (sky-blue) PCM. The overall spectra were shifted by $+4700 \mathrm{~cm}^{-1}$ for without PCM and $+4150 \mathrm{~cm}^{-1}$ for with PCM. 

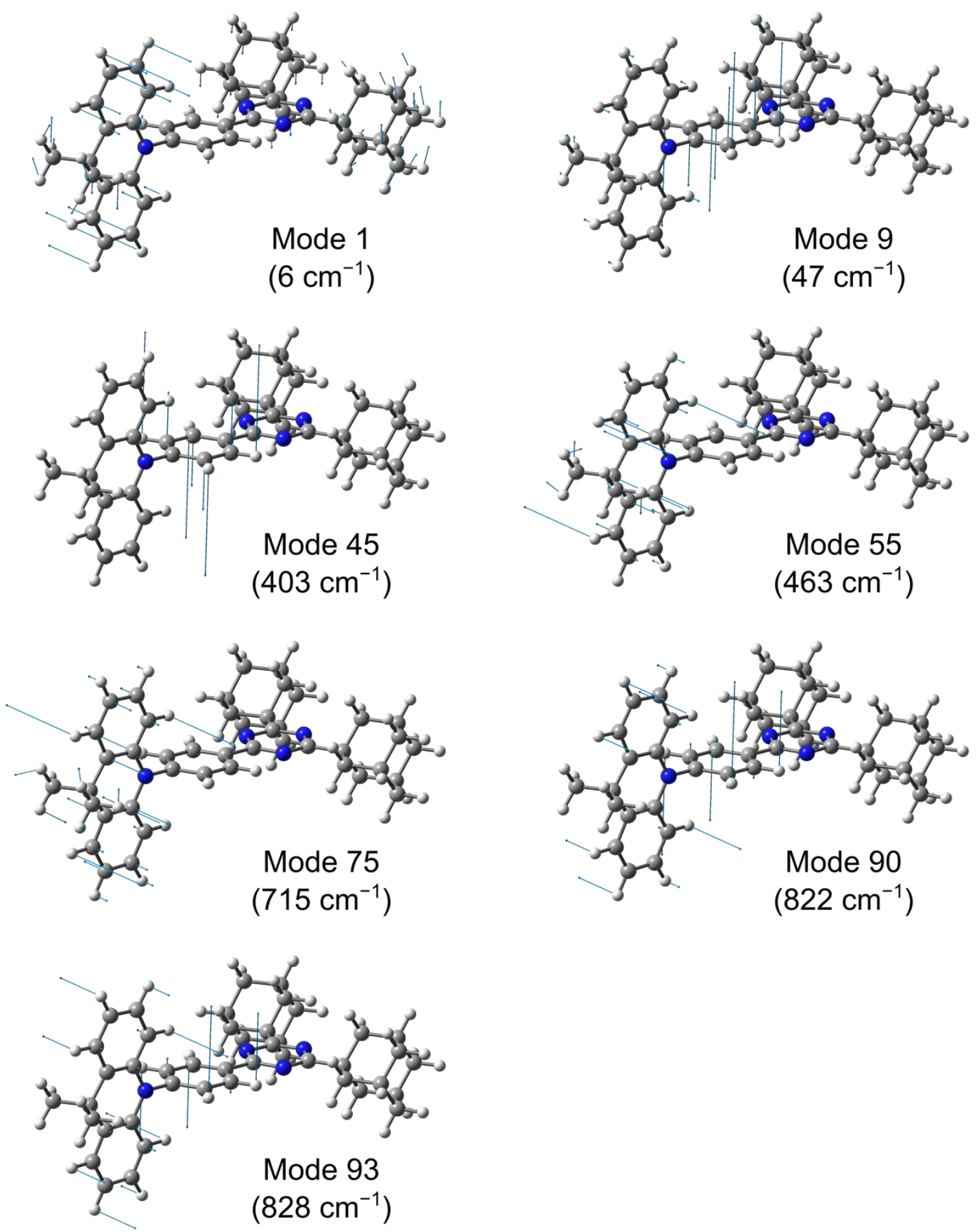

Figure S2. Vibrational modes exhibiting strong transition dipole moment for $\mathrm{C}_{1}$ calculated using PBE0/6-31G(d) functional and basis set. 
(a) $\mathrm{C}_{2}$

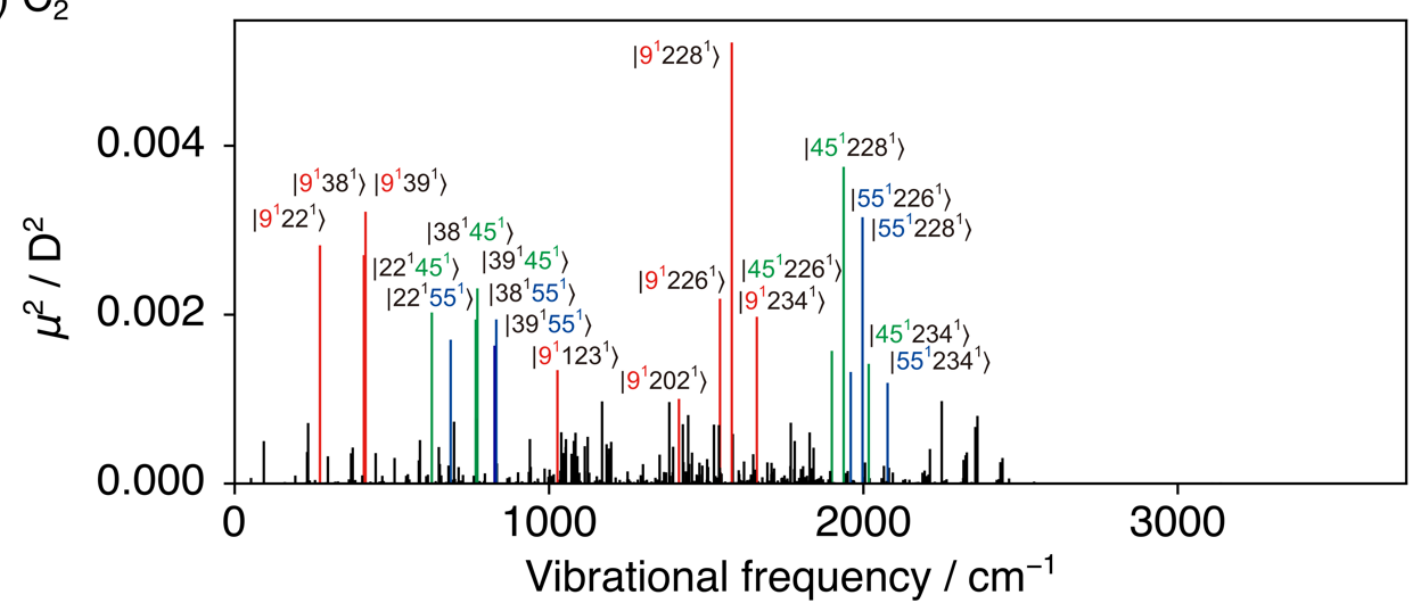

(b) $\mathrm{C}_{3}$

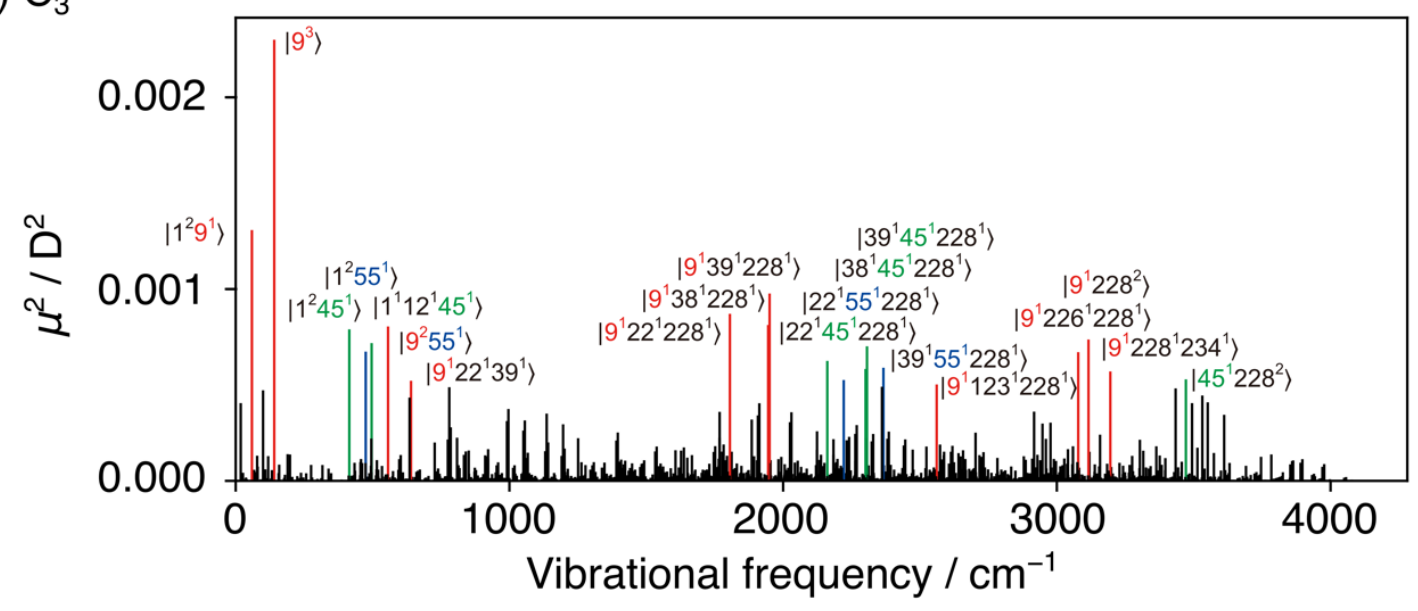

Figure S3. Vibrational modes exhibiting strong transition dipole moment for $\mathrm{C}_{2}$ and $\mathrm{C}_{3}$. Vibrational mode containing mode 9, 45, 55 are colored in red, green, and blue, respectively. For exception, the mode of $\left|9^{2} 55^{1}\right\rangle$ is colored in red although the mode is composed of both mode 9 and mode 55 . 


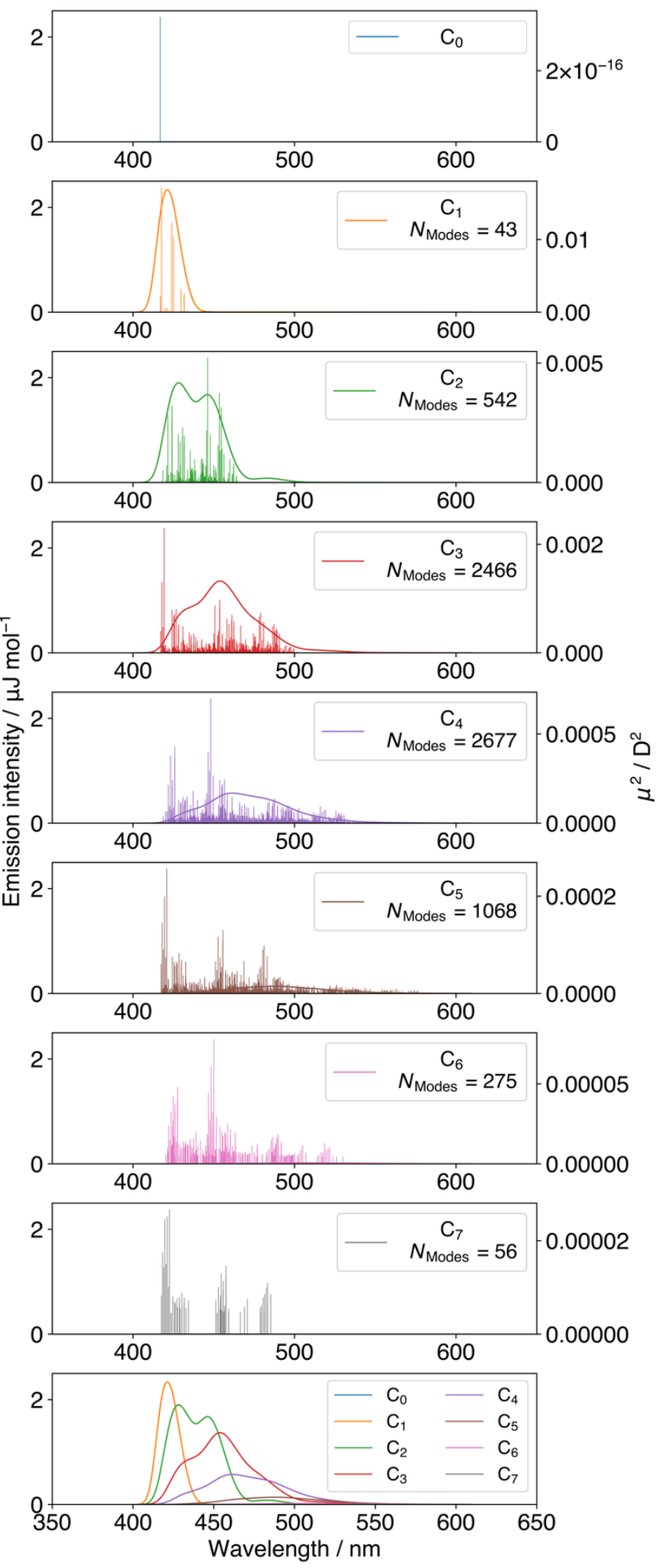

Figure S4. Class-specific spectra (curve lines, left axis) and $\mu^{2}$ of vibronic transitions (vertical lines, right axis) are exhibited from $\mathrm{C}_{0}$ to $\mathrm{C}_{7}$. The vertical lines are enlarged compared to Figure 2 to clearly show respective vibrionic transitions. 


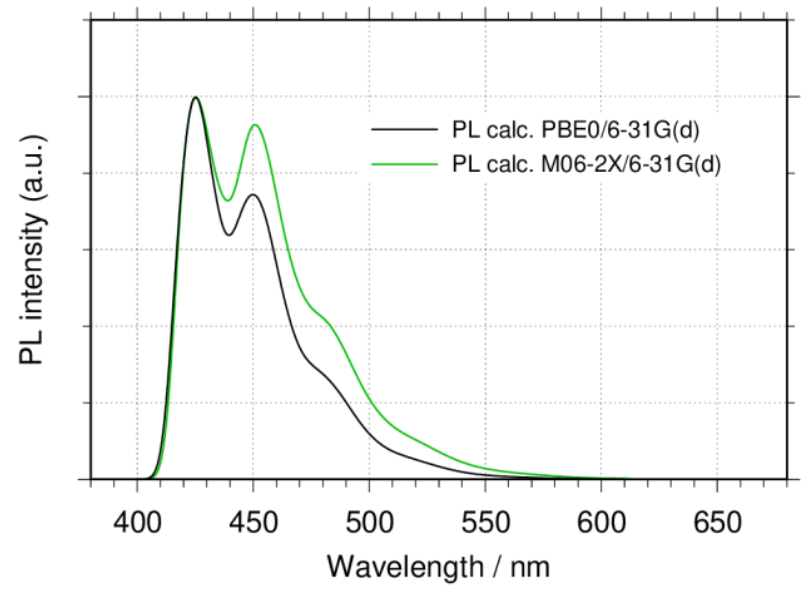

Figure S5. Calculated vibronic spectra of MA-TA for $\mathrm{S}_{1}-\mathrm{S}_{0}$ transition using PBE0/6-31G(d) (black) and M06-2X/6-31G(d) (green). The overall spectra were shifted by $+4700 \mathrm{~cm}^{-1}$ for PBE0/6-31G(d) and $-1850 \mathrm{~cm}^{-1}$ for M06-2X/6-31G(d). 
(a)
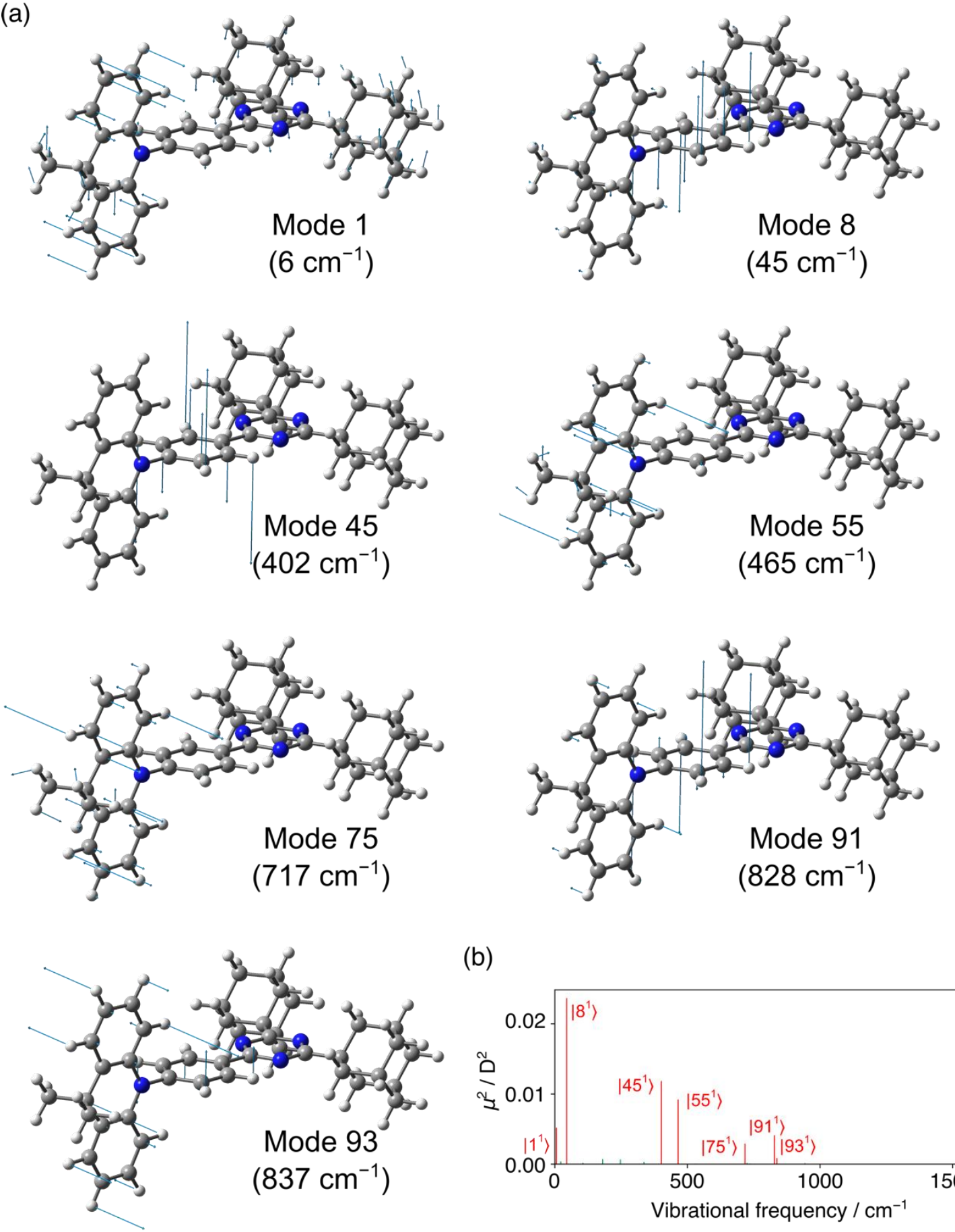

(b)

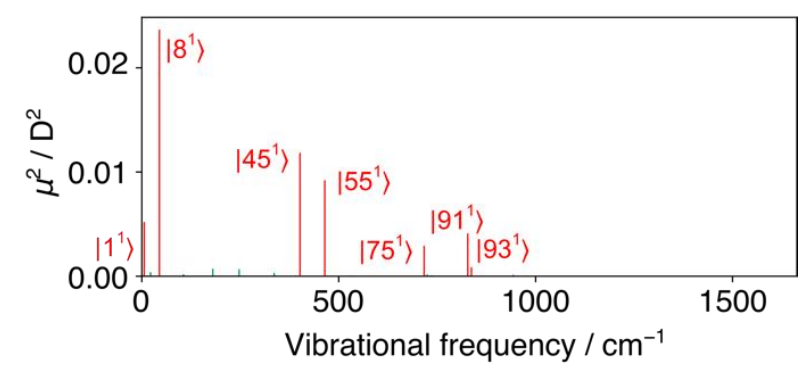

Figure S6. (a) Vibrational modes exhibiting strong transition dipole moment in $\mathrm{C}_{1}$ and (b) $\mu^{2}$ values of vibronic transitions in $C_{1}$ as a function of vibrational frequency, calculated using M06-2X/6-31G(d) functional and basis set. Vibrational modes providing a $\mu^{2}$ value $>0.001$ (except for $\left.\left|93^{1}\right\rangle\right)$ are shown in red and weaker modes are shown in green in (b). 


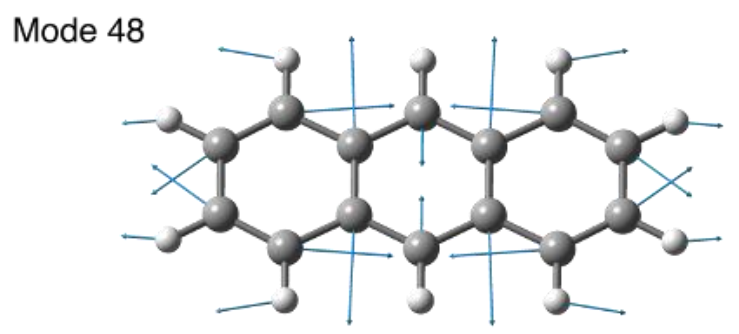

Figure S7. The vibrational mode $48\left(1404 \mathrm{~cm}^{-1}\right)$ exhibiting the strongest transition dipole moment in $\mathrm{C}_{1}$ for anthracene. $\mathrm{PBE} 0 / 6-31 \mathrm{G}(\mathrm{d})$ was used and scaling factor for the vibrational frequency was 0.95 .

\section{REFERENCES}

1. NIST Computational Chemistry Comparison and Benchmark Database, NIST Standard Reference Database Number 101, Release 21, August 2020, Editor: Russell D. Johnson III, http://cccbdb.nist.gov/, DOI:10.18434/T47C7Z, (accessed 4, Mar 2021) 